\title{
Evaluación de la deglución en pacientes con cáncer precoz de laringe tratados con cirugía o radioterapia
}

\author{
Swallowing assessment in early laryngeal cancer patients treated either \\ with surgery or radiotherapy
}

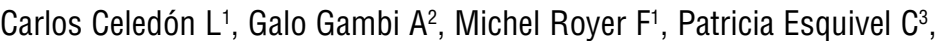 \\ Patricia Arteaga J, Constanza Valdés $\mathrm{P}^{1}$.
}

\begin{abstract}
RESUMEN
Introducción: La deglución es un proceso neuromuscular complejo, que requiere de indemnidad anatómica y de una adecuada coordinación de varios órganos. 1 tratamiento del cáncer laríngeo puede ocasionar trastornos de la deglución. Tradicionalmente se ha reportado una mayor frecuencia de esta alteración con los tratamientos quirúrgicos, sin embargo no existe información adecuada sobre su incidencia en pacientes sometidos a radioterapia por cáncer laríngeo precoz

Objetivo: Comparar la frecuencia de alteraciones de deglución postratamiento en cáncer laríngeo precoz

Material y método: $\theta$ presente es un estudio transversal de dos grupos de pacientes con cáncer laríngeo precoz, unos tratados con cirugía parcial vertical (CP) y otros con radioterapia exclusiva (RT). A cada paciente se le realizó una evaluación otorrinolaringológica, nasofibroscópica y videofluoroscópica postratamiento. Las diferencias entre los grupos se compararon utilizando el test $X$ cuadrado.

Resultados: Ingresaron veinte pacientes por grupo, la mayoría de género masculino y de edades similares. Ambos grupos presentan una alta incidencia de síntomas aspirativos ( $55 \%$ en RT y $35 \%$ en CP) y alteraciones en nasofibroscopía y videofluoroscopía (35\% en RT y $30 \%$ en CP). No hubo diferencias significativas entre ambos grupos.

Discusión y conclusiones: Se demuestra una elevada incidencia de trastorno de la deglución en pacientes tratados por cáncer precoz de laringe, debiendo considerarse como una alteración frecuente en este grupo de pacientes, ya hayan sido tratados con RT o CP.

Palabras claves: Cáncer, laringe, radioterapia, cirugía, deglución.
\end{abstract}

\begin{abstract}
Introduction. Swallowing is a complex neuromuscular process that requires anatomical indemnity and an adequate coordination of several organs. Laryngeal cancer treatment may cause swallowing disorders. Traditionally, a high frequency of this type of disorder after surgery has been reported, but no actual data concerning its incidence in patients undergoing radiotherapy for early laryngeal cancer has been published.

Aim. To compare swallowing disorders frequency posterior to treatment in early laryngeal cancer patients.
\end{abstract}

\footnotetext{
Médico Otorrinolaringólogo, Hospital Clínico Universidad de Chile.

Médico Radioterapeuta, Instituto de Radiomedicina IRAM.

Médico Otorrinolaringóloga, Hospital Barros Luco Trudeau.
} 
Material and Method. Two groups of early laryngeal cancer patients were transversally studied, one treated with vertical partial surgery (CP), and the other treated exclusively with radiotherapy. Each patient had otorhinolaryngological, nasofibroscopic and videofluoroscopic evaluations after treatment. Differences between groups were compared using the_square test.

Results. Twenty patients per group were entered in this study, predominantly males of similar age. Both groups presented a high incidence of aspiration symptoms $155 \%$ in $R T$ and $35 \%$ in CP). There were no significant differences between both groups.

Discussion and Conclusion. A high incidence of swallowing disorders in patients treated for early laryngeal cancer was found. It should then be considered as a frequent alteration in this group of patients, either treated with RT or CP.

Key words: Cancer, larynx, radiotherapy, surgery, swallowing

\section{INTRODUCCIÓN}

El ser humano desde la etapa embrionaria cuenta con un conjunto de estructuras anatómicas genética y congénitamente determinadas, y con una serie de procesos fisiológicos originados en actos reflejos destinados a la preservación de la vida. Entre estos actos se encuentra la deglución, definida como una actividad neuromuscular, producto de una serie de movimientos coordinados de distintos grupos musculares de la cavidad bucal, faringe y esófago, cuyo fin último es el permitir que los líquidos y alimentos sólidos sean transportados desde la boca hacia el estómago.

La deglución es un proceso complejo que requiere de la indemnidad anatómica y funcional de múltiples órganos. Por ello, los pacientes que presentan tumores de vía aerodigestiva refieren dentro de su cuadro clínico la presencia de disfagia ${ }^{1,2}$. El cáncer de laringe es uno de los más frecuentes en cabeza y cuello, excluyendo a los carcinomas derivados de la piel|3,4. Es una patología en que sus principales factores de riesgo son conocidos, tales como el tabaquismo $0^{4,5}$ y el consumo de alcohol ${ }^{6,7} ;$ y porque el tratamiento, pese a ser exitoso puede afectar la fonación, deglución y respiración, alterando de este modo la calidad de vida de los pacientes.

El cáncer laríngeo en su etapa precoz se encuentra definida por los estadios I y II de la clasificación del American Joint Committee on Cancer Staging $(A J C C)^{8}$, en los cuales la presencia del tumor no provoca fijación cordal, no compromete cartílago ni presenta enfermedad ganglionar.
Las modalidades de tratamiento descritas son la radioterapia, la cirugía parcial de laringe y la cirugía transoral con láser, con resultados similares en cuanto a tasas de sobrevida y conservación laríngea ${ }^{9-11}$. Por lo tanto, la elección de una alternativa terapéutica no sólo está determinada por la extensión del tumor sino debe considerar otros factores como la condición general del paciente, su actividad laboral, su calidad vocal, entre otras.

En pacientes con cáncer precoz de laringe, tradicionalmente se ha descrito una mejor calidad vocal postratamiento en aquellos pacientes que recibieron radioterapia en comparación a los pacientes sometidos a cirugía parcial de laringe. El establecimiento del láser como herramienta terapéutica ha demostrado resultados similares a la radioterapia en cuanto a calidad vocal ${ }^{12}$.

Se ha descrito una mayor alteración de la deglución en los pacientes sometidos a cirugía parcial de laringe que aquellos tratados con radioterapia como alternativa única de tratamiento. En general se acepta que las técnicas de cirugía parcial, horizontal (supraglótica) y vertical (frontolateral), ocasionan trastornos de la deglución en el posoperatorio inmediato, pero que durante la evolución existe una mejoría clínica, que no debiera dejar mayores secuelas ${ }^{13}$. Por su parte, los pacientes que reciben radioterapia presentan alteraciones de la deglución, descritas como transitorias, relacionadas principalmente a la aparición de mucositis. En la práctica clínica se observa que un número no determinado de pacientes con cáncer laríngeo precoz tratado con radioterapia 0 cirugía parcial de laringe refieren tos $y / 0$ "atoros" en 
relación a la ingesta de líquidos por plazos prolongados.

Debe considerarse que las técnicas de cirugía parcial de laringe conllevan modificaciones de su configuración anatómica debido a la resección parcial de su musculatura y fibrosis parcial del resto de la laringe, lo que lleva a debilidad de una de las estructuras relevantes en la deglución, como es la laringe $\mathrm{e}^{14,15}$. En tanto, la radioterapia puede afectar a la deglución por sus efectos adversos sobre los tejidos, causando no sólo mucositis, sino también fibrosis, alteraciones en la motilidad, falta de sensibilidad y xerostomía. Además, las evaluaciones en pacientes con cáncer avanzado de laringe tratados con quimiorradioterapia han demostrado alteraciones significativas en la deglución y calidad vocal ${ }^{16-19}$. No existe en la literatura internacional mucha información respecto a deglución posradioterapia en pacientes con cáncer precoz de laringe, por lo que parece necesario precisar las impresiones clínicas estudiando la presencia de alteraciones de la deglución en pacientes con cáncer precoz de laringe tratados con RT y CP.

La hipótesis de trabajo es que en los estadios precoces de cáncer laríngeo, tanto en los pacientes tratados con cirugía parcial como en los tratados con radioterapia exclusiva, se produce igualmente trastorno de la deglución crónica.

\section{MATERIAL Y MÉTODO}

Se realiza un estudio transversal de pacientes con cáncer precoz de laringe durante los años 2004 a 2006. Durante este período se planifica y se realiza el estudio que incluyen aquellos pacientes con diagnóstico de cáncer precoz de laringe, estadios I y II (AJCC) ${ }^{8}$, tratados con cirugía parcial o radioterapia entre los años 1994 y 2004 en el Hospital Clínico de la Universidad de Chile. Se excluyen pacientes con recidiva tumoral, cirugías laríngeas previas al tratamiento indicado, enfermedad neuromuscular 0 digestiva conocida.

Ingresan al estudio cuarenta pacientes tratados por cáncer precoz de laringe, los que se dividen en dos grupos para su análisis: 20 se sometieron a una cirugía parcial de laringe frontolateral (CP) y 20 recibieron radioterapia (RT) como modalidad única de tratamiento.
Para cada paciente se confecciona un formulario individual, con su identificación y los antecedentes del tratamiento (técnica quirúrgica; dosis, campo y duración de radioterapia). Igualmente en esta ficha se consigna una evaluación clínica de la deglución, que comprende historia clínica (vía de alimentación, síntomas aspirativos durante la alimentación, infecciones pulmonares, etc.) y hallazgos al examen físico (movilidad de paladar y lengua, retención de saliva en hipofaringe o laringe, aspiración, etc.). Se registra además la nasofibroscopía, en la cual se constata: retardo en motilidad faríngea, penetración, aspiración y residuo de saliva o alimento; y la videofluoroscopía, en la cual cada sujeto recibe dos consistencias de bario (líquida y semisólida). Esta información se consigna con videocámara de $8 \mathrm{~mm}$. Posteriormente se revisan los videos para determinar los siguientes parámetros: tiempo de tránsito faríngeo (que comprende el movimiento del bolo desde la rama de mandíbula a la región cricofaríngea), cierre aritenoepiglótico (tiempo de contacto entre aritenoides y base de epiglotis), elevación del hioides, función del esfínter esofágico superior (tiempo en que permanece abierto el esfínter cricofaríngeo durante la deglución) y aspiración.

La evaluación, tanto clínica, nasofibroscópica como videofluoroscópica se realizaron en todos los pacientes, por lo menos seis meses postratamiento

Se considera como patrón de normalidad el valor publicado por Cuadra y col..$^{20}$ en tesis de grado de la carrera de fonoaudiología realizado sobre parámetros de videofluroscopía en adultos normales.

Para el análisis estadístico se calcula un tamaño muestral de dos grupos de veinte pacientes cada uno, y se utiliza el test de Xi-cuadrado considerándose significativo un $p$ igual o menor a 0,05.

\section{RESULTADOS}

De los cuarenta pacientes tratados por cáncer precoz de laringe entre los años 1994 a 2004 y que se dividieron en dos grupos para su análisis: 20 se sometieron a una cirugía parcial de laringe frontolateral (CP) y 20 que recibieron radioterapia (RT) como modalidad única de tratamiento; el género masculino predomina en ambos grupos y 
las edades promedios se encuentran sobre los sesenta años (Tabla 1).

En la evaluación clínica realizada, el $100 \%$ de los pacientes recibe alimentación vía oral, pero destaca la alta presencia de síntomas aspirativos referidos por los pacientes (tales como "atoros", "tos" o "trapicarse" al comer), la que supera el $30 \%$ en ambos grupos (Tabla 2).

En cuanto a los hallazgos en la nasofibroscopía, se presentan alteraciones en cuatro (CP) y siete pacientes (RT), caracterizados por penetración y/o aspiración de alimentos o saliva.

La videofluoroscopía detectó alteraciones en seis (CP) y siete pacientes (RT). La diferencia de alteraciones entre los grupos no es estadísticamente significativa $(p>0,05)$. De los parámetros analizados la elevación del hioides y el cierre de los pliegues aritenoepiglóticos fueron en los que se detectaron alteraciones con mayor frecuencia en ambos grupos (Gráfico 1).

\section{DISCUSIÓN}

La deglución es un proceso neuromuscular complejo que requiere una integridad anatómica de varios órganos y una adecuada coordinación funcional entre ellos. Debido a esta complejidad el tratamiento del cáncer laríngeo se acompaña de alteraciones en la deglución. Este punto, que está demostrado en los diversos esquemas terapéuticos para etapas avanzadas de cáncer laríngeo, no ha sido explorado consistentemente en sus etapas precoces. Existe información suficiente sobre los trastornos de la deglución aguda producida en la cirugía parcial de laringe, pero no existe información suficiente sobre los trastornos crónicos de la deglución. Con respecto a los pacientes con cáncer precoz de laringe y que reciben radioterapia como tratamiento exclusivo, no hay estudios suficientes tanto de sus trastornos de la deglución aguda y mucho menos en la crónica.

En nuestra experiencia clínica observamos que en los pacientes con cáncer precoz de laringe sometidos a tratamiento sea radiante 0 quirúrgico se producían alteraciones crónicas de la deglución. Además, los efectos adversos conocidos de la radioterapia sobre los tejidos y los reportes de alteraciones de deglución en pacientes que recibieron quimiorradioterapia para cáncer avanzado ${ }^{16-19}$, nos motivaron a estudiar la real frecuencia de este problema en pacientes con cáncer laríngeo precoz tratado.

Tabla 1. Pacientes con cáncer precoz de laringe tratados con radioterapia o cirugía parcial

\begin{tabular}{|lcc|}
\hline & Radioterapia & Cirugía parcial \\
\hline Edad (Rango) & $68(54-86)$ & $60(49-73)$ \\
Género masculino & $85 \%$ & $95 \%$ \\
Tabaquismo & $45 \%$ & $70 \%$ \\
T1 & $80 \%$ & $70 \%$ \\
T2 & $20 \%$ & $30 \%$ \\
\hline
\end{tabular}

Tabla 2. Evaluación clínica de alteraciones de deglución en pacientes con cáncer precoz de laringe tratados con radioterapia o cirugía parcial

\begin{tabular}{|lcc|}
\hline & Radioterapia & Cirugía parcial \\
\hline Alimentación vía oral & $100 \%$ & $100 \%$ \\
Síntomas aspirativos & $55 \%$ & $35 \%$ \\
Neumonía aspirativa & $15 \%$ & $0 \%$ \\
Alteración movilidad paladar & $5 \%$ & $0 \%$ \\
Alteración movilidad lengua & $5 \%$ & $0 \%$ \\
Alteración manejo saliva & $20 \%$ & $0 \%$ \\
\hline
\end{tabular}




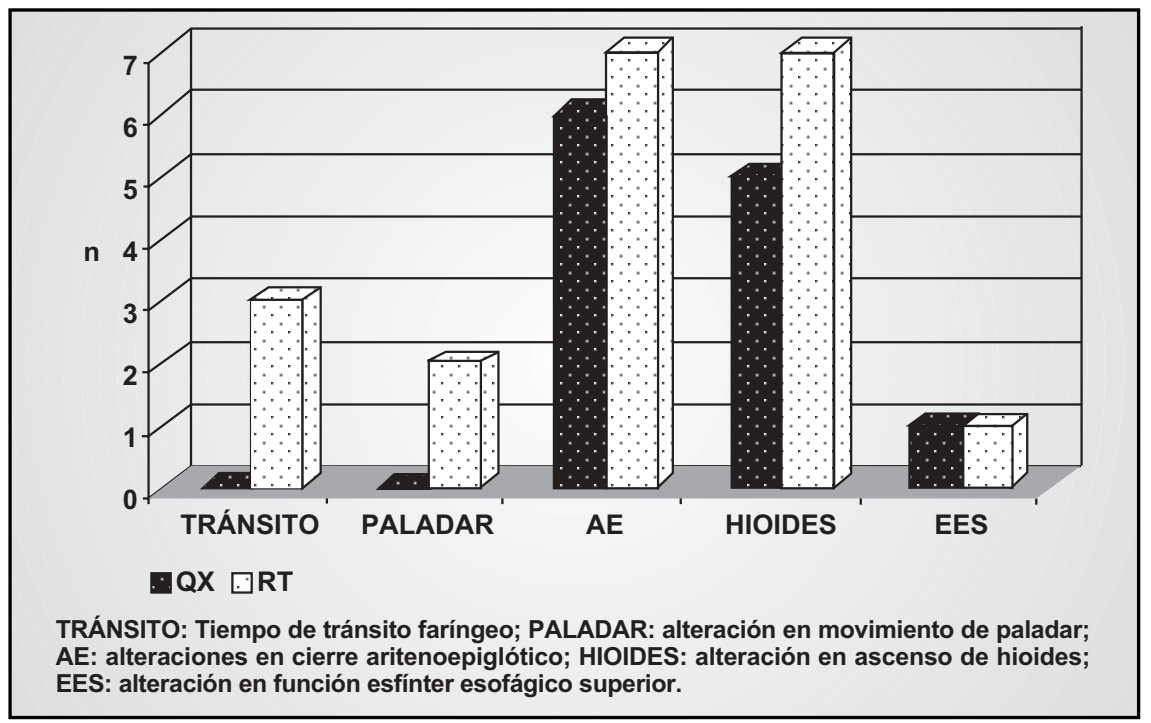

Gráfico 1: Alteraciones en videofluoroscopía en pacientes con cáncer precoz de laringe tratados con radioterapia o cirugía parcial

La elección de la alternativa de tratamiento frente a un cáncer precoz de laringe debe ser una alternativa unimodal del primario, sin necesidad de realizar un tratamiento adicional al cuello. Como conducta general del Servicio ORL del Hospital Clínico de la Universidad de Chile; los tumores de cuerda clasificados como $\mathrm{T} 1$ han recibido radioterapia exclusiva sobre ambas cuerdas vocales. Si existe compromiso de la comisura anterior 0 el tumor es clasificado como T2 se recomienda la cirugía parcial de laringe como alternativa terapéutica.

Esta serie de pacientes con cáncer laríngeo precoz demuestra la existencia de alteraciones de la deglución posteriores al tratamiento, tanto en sujetos sometidos a cirugía parcial (laringectomía frontolateral) como aquellos sometidos a radioterapia. Debe destacarse que todos los pacientes mantienen alimentación vía oral, y en ambos grupos hay pacientes que refieren síntomas aspirativos durante la alimentación, pero con escasa limitación funcional y sin complicaciones relacionadas, siendo la frecuencia de neumonías durante el seguimiento muy baja (datos no mostrados). Tanto en la evaluación clínica, nasofibroscópica y videofluoroscópica, se evidencian alteraciones en ambos grupos, sin existir diferencias estadísticamente significativas.
En el análisis debe recordarse que los resultados de esta serie son postratamiento y ninguno de ellos tenía alteración de la deglución previo al tratamiento. Se ha descrito que los pacientes con cáncer de cabeza y cuello pueden presentar alteraciones deglutorias previas a su tratamiento, pero los tumores precoces y de ubicación laríngea y concretamente de cuerda vocal, presentan una menor frecuencia de alteraciones que tumores de otras localizaciones y/0 estadios avanzados ${ }^{21,22}$. Además nuestra serie presenta con mayor frecuencia la alteración del cierre aritenoepiglótico; lo que difiere de lo descrito por Pauloski, de una prolongación del tránsito faríngeo, un mayor porcentaje de residuo y alteración en la función del esfínter esofágico superior, pero manteniendo un adecuado cierre aritenoepiglótico ${ }^{21}$.

Consideramos que los resultados son relevantes, ya que demuestran que frente a pacientes tratados por cáncer precoz de laringe, con una u otra modalidad, es frecuente encontrar trastornos de la deglución crónicos. Los pacientes que han sido tratados por cáncer laríngeo precoz, sea con CP o RX tendrán con los años un mayor déficit laríngeo en comparación con pacientes sin tratamiento, ya que éste se sumaría al déficit funcional laríngeo propio de la edad. En efecto, en los pacientes añosos uno de los problemas de salud 
que presentan es el trastorno de la deglución, y si a esto se suma el déficit funcional laríngeo secundario a su tratamiento del cáncer laríngeo precoz, se tendría la explicación de algunas de las neumonías que hemos observado en la clínica de algunos de estos pacientes. ¿Serán todas o la mayoría de estas neumonías secundarias a aspiración como se constató en algunos de nuestros pacientes? Comprobar esta hipótesis sería materia de otra investigación prospectiva.

Debemos mencionar que en este estudio no se incluye la cirugía transoral con láser, pero es de suponer que una cordectomía por láser desde el punto de vista funcional laríngeo debería tener gran diferencia.

La presente serie incluye pacientes evaluados a diferentes plazos postratamiento. Ninguno de ellos antes de los 6 meses postratamiento. Esto representa un riesgo, dado que otros factores pudieran influir en la aparición de disfagia, pero como la finalidad del trabajo era determinar la frecuencia de alteraciones de la deglución permanentes postratamiento y no el efecto inmediato de cirugía 0 radioterapia, nos parece que este factor tiempo no invalidan los resultados obtenidos.

En suma, consideramos importante demostrar que posterior al tratamiento del cáncer precoz de laringe, con radioterapia o cirugía parcial, existe una alta frecuencia de trastornos de la deglución, sin existir diferencias significativas entre ambas modalidades terapéuticas.

Trabajo financiado por la OAIC-HCUCH. El proyecto recibió la aprobación para su realización por parte del Comité de Ética del Hospital Clínico de la Universidad de Chile ( $\mathrm{HCUCH})$ y fue financiado por la Oficina de Apoyo a la Investigación Clínica (OAIC).

\section{BIBLIOGRAFÍA}

1. Morton RP. A profile of laryngeal cancer in Auckland 1965-1979. NZMed J1982; 95: 652-5.

2. Merletti $F$, Faggiano $F$, Boffetta $P$, et al. Topographic classification clinical characteristics and diagnostic delay of cancer of the larynx/hypopharynx in Torino Italy. Cancer 1990; 66: 1711-6.

3. Ferlito A. The natural history of early vocal cord cancer. Acta Otolaryngol 1995; 115(2): 345-7.
4. Koufman JA, Burke $A$. The etiology and pathogenesis of laryngeal carcinoma. Colaryngologic Cin North Am 1997; 30(1): 1-19.

5. Falk RT, PICKLe LW, Brown LM, Mason TJ, Buffler PA, FraumenI JF JR. Effect of smoking and alcohol consumption on laryngeal cancer risk in coastal Texas. Cancer Res 1989; 49: 4024-9.

6. Guenel P. A study of the interaction of alcohol drinking and tobacco smoking among French cases of laryngeal cancer. $J$ Epidemiol Community Health 1988; 42(4): 350-4.

7. PICCIRILLo JF. Importance of comorbidity in head and neck cancer. Laryngoscope 2000; 110(4): 593-602.

8. Greene F et al, eds. Larynx. En: AJCC Cancer Staging Manual. Philadelphia, Pa: Lippincott Raven Publishers, Sexta edición, 2002, 47-58.

9. Jones AS, Fish B, Fenton JE, Husband DJ. The treatment of early laryngeal cancers (T1-T2 N0): surgery or irradiation? Head Neck 2004; 26(2): 127-35.

10. BACK G, Sood S. The management of early laryngeal cancer: options for patients and therapists. Ourr Ooin Otolaryngol Head Neck Surg 2005; 13(2): 85-91.

11. Cragle SP, BrandenbuRg J. Laser cordectomy or radiotherapy: cure rates, communication, and cost. Otolaryngol Head Neck Surg 1993; 108(6): 648-54.

12. Spector JG, Sessions DG, Chao KS et al. Stage I (T1 NO MO) squamous cell carcinoma of the laryngeal glottis: therapeutic results and voice preservation. Head Neck 1999; 21(8): 707-17.

13. Rademaker AW, Logemann Ja, Pauloski BR, et al. Recovery of postoperative swallowing in patients undergoing partial laryngectomy. Head Neck 1993; 15: 325-34.

14. Logemann J. Swallowing physiology and pathophysiology. Aolaryngol Cin North Am 1988; 21: 613-23. 1990; 66: 1711-6.

15. Logemann J. Mechanisms of normal and abnormal swallowing. En: Cummings CW, Fredrickson JM, Harker LA. Eds. Otolaryngology-Head and Neck Surgery, Vol. 3. Mosby: St. Louis, 1998, pp 1844-53.

16. Lazarus CL, Logemann JA, Pauloski BR, et al. Swallowing disorders in head and neck cancer patients treated with radiotherapy and adjuvant chemotherapy. Laryngoscope 1996; 160: 1157-66. 
17. Smith RV, Kotz T, Beitler JJ, Wadler S. Long-term swallowing problems after organ preservation therapy with concomitant radiation therapy and intravenous hydroxyurea. Arch Otolaryngol Head Neck Surg 2000; 126: 384-9.

18. Kotz T, Costello R, Li Y, Posner MR. Swallowing dysfunction after chemoradiation for advanced squamous cell carcinoma of the head and neck. Head Neck 2004; 26(4): 365-72.

19. Dworkin JP, Hill SL, Stachler RJ, Meleca RJ, Kewson D. Swallowing Function Outcomes Following Nonsurgical Therapy for AdvancedStage Laryngeal Carcinoma Dysphagia 2006; 21(1): 66-74.
20. Cuadra P, Feliú P, González G, González J, Pinto P. Análisis descriptivo de los patrones de deglución en un grupo de adultos normales. Seminario de Investigación, Escuela de Fonoaudiología, Universidad de Chile, 1996.

21. Pauloski BR, Rademaker aW, Logemann Ja et al. Pretreatment swallowing function in patients with head and neck cancer. Head Neck 2000; 22(5): 474-82.

22. Stenson KM, MacCracken E, List M et al. Swallowing function in patients with head and neck cancer prior to treatment. Arch Otolaryngol Head Neck Surg 2000; 126: 371-7. 